\title{
PHARMACOKINETICS AND BIODISTRIBUTION STUDIES OF DOXORUBICIN LOADED POLY(BUTYL CYANOACRYLATE) NANOPARTICLES SYNTHESIZED BY TWO DIFFERENT TECHNIQUES
}

\author{
L. Harivardhan Reddy and R. S. R. Murthy*
}

Drug Delivery Research Laboratory, Center of Relevance and Excellence in NDDS, Pharmacy Department, G.H. Patel Building, Donor's Plaza, M.S. University, Fatehgunj, Baroda-390002, Gujarat, India

e-mail:m_rsr@rediffmail.com

Received: May 10, 2004; Accepted: August 5, 2004

Key words: Poly(butyl cyanoacrylate)/Nanoparticle/Emulsion polymerization/Long circulating carrier/Pharmacokinetics/ Biodistribution

The aim of the study is to determine and compare the pharmacokinetics and tissue distribution of Doxorubicin (Dox) delivered as solution or through nanoparticles after intravenous (i.v.) and intraperitoneal (i.p.) injection. Doxorubicin loaded poly(butyl cyanoacrylate) nanoparticles were synthesized by dispersion polymerization (DP) and emulsion polymerization (EP) techniques. The drug loaded DP and EP nanoparticles were administered by i.v. or i.p. routes and the respective pharmacokinetics and tissue distribution were determined. Both types of nanoparticles significantly enhanced the elimination half-life $\left(\mathrm{T}_{1 / 2}\right)$, mean residence time (MRT) $\mathrm{AUC}^{0-8}, \mathrm{AUC}^{0-\infty}$ and $\mathrm{AUMC}^{0-8}$ of Dox in blood after i.v. injection. Dox delivered through DP nanoparticles rapidly disappeared from blood and distributed to the organs of reticuloendothelial system (RES). But, the clearance of Dox delivered through EP nanoparticles from blood was slower than this of the DP nanoparticles and Dox solution. After i.p. injection, the Dox loaded into DP nanoparticles quickly appeared in blood and undergone rapid distribution to the organs of RES, while the Dox loaded into EP nanoparticles absorbed slowly into blood and remained in the circulation for longer time. The absorption into blood of Dox delivered through DP and EP nanoparticles after i.p. injection was relatively rapid and higher than Dox solution. The $\mathrm{T}_{1 / 2}, \mathrm{MRT}, \mathrm{AUC}^{0-8}, \mathrm{AUC}^{0-\infty}$ and $\mathrm{AUMC}^{0-8}$ of Dox in blood were significantly higher and the clearance $(\mathrm{Cl})$ was lower than for the Dox solution after i.p. injection. The tissue concentrations of Dox delivered through nanoparticles after i.p. injection were significantly lower than after i.v. injection. The bioavailability (F) of Dox was greatly enhanced by DP ( 1.9 fold) and EP nanoparticles ( 2.12 fold $)$ compared to Dox solution after i.p. injection. EP nanoparticles significantly enhanced the bioavailability, MRT, $\mathrm{T}_{1 / 2}, \mathrm{AUC}^{0-8}, \mathrm{AUC}^{0-\infty}$ and $\mathrm{AUMC}^{0-8}$ of Dox than DP nanoparticles. This signifies the advantage of EP nanoparticles in increasing the elimination half-life of Dox both after i.v. and i.p. injection and enhanced bioavailability after i.p. injection, which is expected to improve the therapeutic efficacy of Dox and reduce the Dox-associated systemic toxicity. Importantly, both DP and EP nanoparticles greatly reduced the distribution of Dox to heart both after i.v. and i.p. injection, suggesting their potential in reducing Dox-associated cardiotoxicity.

\section{INTRODUCTION}

Development of efficient drug delivery systems has attracted great attention during the last two decades. The delivery of drug molecules through the carrier systems is assumed to avoid their unwanted effects because of controlled biodistribution ${ }^{1}$. A considerable attention has been observed in the recent years in the use of nanoparticles as drug carriers due to their stability, biodegradability and ease of preparation. ${ }^{2}$ Polybutyl cyanoacrylate (PBC) nanoparticles have gained increasing interest in the drug delivery for pharmaceutical and medical applications. These nanoparticles alter the body distribution of incorporated drugs ${ }^{3}$, protect the drugs against enzymatic degradation ${ }^{4}$, and reduce the toxic effects of drug molecules. ${ }^{5}$ Majority of the earlier studies reported the dispersion polymerization technique for the synthesis of
PBC nanoparticles. ${ }^{6-8}$ We have recently reported the synthesis of PBC nanoparticles of smaller size by emulsion polymerization technique. ${ }^{9}$

A major limiting factor to the systemic use of particulate delivery systems is the rapid clearance of carrier from the blood circulation by reticuloendothelial system (RES). Various techniques such as suppression of RES ${ }^{10}$ and modification of surface characteristics of drug carriers by coating with block copolymers ${ }^{11}$ were attempted to reduce the RES uptake. The second approach has been shown to be highly effective in altering the biodistribution pattern of colloidal drug carriers. ${ }^{12}$ Recently long circulating nanoparticles were obtained by surface modification with dysopsonic polymers such as poly(ethylene glycol). ${ }^{13}$

Polycyanoacrylate nanoparticles were recently used for drug targeting to brain ${ }^{14}$, spleen ${ }^{15}$ and intestinal epithelium. ${ }^{16}$ Majority of the above targeting approaches 
utilized surface modification mechanisms. Gulyaev et al. ${ }^{14}$ coated doxorubicin loaded poly(butyl cyanoacrylate nanoparticles with polysorbate 80 to enhance the brain transport, while Peracchia et al. ${ }^{15}$ performed PEGylation of cyanoacrylate for splenic targeting. For targeting of particulate systems to cancers, recently an important mechanism called enhanced permeability and retention (EPR) effect was used. ${ }^{17}$ The particles injected into blood enter tumor vasculature and reaches cancer cells via the distribution through vascular compartment, transport across microvascular wall and transport through the interstitial compartment. ${ }^{18}$ Majority of tumors possess leaky vasculature through which the macromolecular carrier systems can penetrate and concentrate in the tumors. ${ }^{19}$ Enhancement of drug transport to tumors by this mechanism necessitates the prolonged circulation of carrier in blood. Thus prolongation of circulation time of $\mathrm{PBC}$ nanoparticles in blood would facilitate controlled tissue distribution, limit the drug associated toxicity and high tumor drug transport by EPR effect.

Doxorubicin hydrochloride is a cytotoxic anthracycline antibiotic widely used in the treatment of non-Hodgkin's lymphoma, acute lymphoblastic leukemia, breast carcinoma and several others types of cancers. The mechanism of cytotoxicity involves the specific intercalation of planar anthracycline nucleus of DH to the DNA double helix resulting in the prevention of further DNA replication. ${ }^{20}$

In the present study, Doxorubicin-loaded Poly(butyl cyanoacrylate) nanoparticles were prepared by dispersion polymerization and emulsion polymerization techniques. The nanoparticles were injected into albino rats by i.v. or i.p. route and their pharmacokinetics and tissue distribution were determined. The paper also describes the influence of type of nanoparticles and of the route of administration on the pharmacokinetics and tissue distribution.

\section{EXPERIMENTAL}

\section{Materials}

n-butyl cyanoacrylate monomer was obtained from Sun Pharmaceutical Industries Limited (India). Doxorubicin $\mathrm{HCl}$ was a kind gift by Claris Life Science, India. Dextran 70 (molecular mass 70,000) was kindly supplied by Claris Life Sciences Limited (India). Poloxamer 188 was purchased from Sigma (USA). All other chemicals used in the study were of analytical grade. Water used in all the studies was distilled and filtered through $0.22 \mu \mathrm{m}$ nylon filter before use.

\section{Synthesis of Poly (butyl cyanoacrylate) (PBC) nanoparticles}

$\mathrm{PBC}$ nanoparticles containing Doxorubicin $\mathrm{HCl}$ (Dox) were synthesized by both dispersion polymerization (DP) and emulsion polymerization (EP) techniques. In DP technique, the cyanoacrylate monomer was added dropwise into the polymerization medium containing Dox and Dextran $70(0.1 \% \mathrm{~m} / \mathrm{V})$ under stirring at $700 \mathrm{rpm}$.
Stirring was continued after monomer addition for $3 \mathrm{~h}$, and then the dispersion was neutralized to $\mathrm{pH} 7.0$ with phosphate buffer containing $\mathrm{NaOH} \mathrm{pH} \mathrm{7.4.}$

Dox loaded PBC nanoparticles were prepared by EP technique as reported earlier. ${ }^{9}$ Briefly, the monomer was added dropwise into the polymerization medium containing Dox and Poloxamer $188(0.5 \% \mathrm{~m} / \mathrm{V})$ and proceeded as for the dispersion polymerization.

\section{Determination of drug content in nanoparticles}

Nanoparticulate dispersion was centrifuged at 15000 RPM for 30 min in a cooling centrifuge (Remi C24, Remi, India). The supernatant was decanted and the sediment was washed thrice with distilled water to separate the free drug from nanoparticles, and freeze-dried. The dried sample was weighed and dissolved in methanol, and estimated for drug content in UV-Visible spectrophotometer (Hitachi U 2000, Japan) at $495 \mathrm{~nm}$.

\section{Particle size measurement}

Particle size measurements were carried out by dynamic light scattering using Malvern Hydro 2000SM particle size analyzer (Malvern instruments, UK). Aqueous nanoparticulate dispersions were injected through sample injection port, and the laser obscuration range was maintained between $10-20 \%$. The analysis was performed thrice and average values were taken.

\section{Pharmacokinetic and biodistribution studies}

All the animal studies were approved by the CPCSEA and local animal ethics committee. The studies were performed in albino rats weighing about 200-250 gm. The rats were fasted overnight before experimentation and were accessed to water ad libitum. The aqueous nanoparticulate dispersion equivalent to $5 \mathrm{mg} / \mathrm{kg}^{14}$ Dox $\mathrm{HCl}$ in saline was administered to rats either by intravenous (i.v.) or intraperitoneal (i.p.) injection. After predetermined time intervals, the rats were sacrificed and organs such as liver, lung, kidney, heart and spleen were isolated. For pharmacokinetic studies, blood was collected from the retro-orbital plexus of rat eye. Estimation of Dox in blood and tissues was performed by fluorimetric estimation ${ }^{21}$ using spectrofluorophotometer (Shimadzu RF-540, Shimadzu Corporation, Japan). The analytical method development is briefed as follows. To $0.1 \mathrm{ml}$ blood or $1 \mathrm{ml}$ tissue homogenate was added the corresponding quantity of stock solution of Dox ranging from $50-1000 \mathrm{ng} / \mathrm{ml}$ for blood, and 100-1000 ng/ml for tissues. The contents were vortexed for $15 \mathrm{sec}$ and kept aside for $30 \mathrm{~min}$. The contents were then treated with $0.5 \mathrm{ml} 5 \%$ TCA solution as protein precipitant and vortexed for $15 \mathrm{sec}$, followed by the addition of $0.3 \mathrm{ml} \mathrm{pH} 9.2$ alkaline borate buffer and mixed for $15 \mathrm{sec}$. The drug was extracted with $1 \mathrm{ml}$ dichloromethane (DCM) by vortexing for $3 \mathrm{~min}$, and centrifuged at 6000 RPM for 4 min and the bottom organic layer was collected. The extraction was repeated twice for aqueous portion with $1 \mathrm{ml} \mathrm{DCM}$ every time. Finally, the extracts were combined and the volume was made up to $3 \mathrm{ml}$ with DCM. The fluorescence of extracted drug was 
measured in spectrofluorophotometer at wavelengths of excitation at $480 \mathrm{~nm}$ and emission at $558 \mathrm{~nm}$.

\section{Statistical analysis}

All the data are reported as mean \pm SD of three experiments. Statistical comparison of the data was done by ANOVA at a significance level of $\mathrm{P}<0.001$, and Student's $\mathrm{t}$-test at a significance level of $\mathrm{P}<0.001$.

\section{RESULTS}

Dox loaded poly(butyl cyanoacrylate) nanoparticles were synthesized by dispersion polymerization and emulsion polymerization techniques. The EP nanoparticles possessed smaller size $(175 \mathrm{~nm})$ than DP nanoparticles (315 nm). The entrapment efficiencies of DP and EP nanoparticles were found to be $66.7 \%$ and $64.5 \%$ respectively at $1 \% \mathrm{v} / \mathrm{v}$ monomer concentration.

Fig. 1 represents the blood clearance profiles of Dox solution and Dox loaded DP and EP nanoparticles after i.v. injection in albino rats. The Dox loaded into DP nanoparticles experienced an initial rapid clearance from the blood, followed by slow clearance after $2 \mathrm{~h}$ of injection. The concentration of Dox delivered through DP nanoparticles after i.v. injection was significantly higher than Dox solution after $2 \mathrm{~h}$ post injection. This is in agreement with the earlier report by Gulyaev at al. ${ }^{14}$ In contrast, the Dox into loaded EP nanoparticles showed higher blood concentrations throughout the study. The pharmacokinetic parameters were determined by analyzing the data by Wagner Nelson method. The parameters after i.v. injection are shown in table 1 . The pharmacokinetics of Dox solution and Dox delivered through DP and EP nanoparticles differed significantly. Both DP and EP nanoparticles significantly increased the half-life $\left(\mathrm{T}_{1 / 2}\right)$ and mean residence time (MRT) of Dox in blood. The area

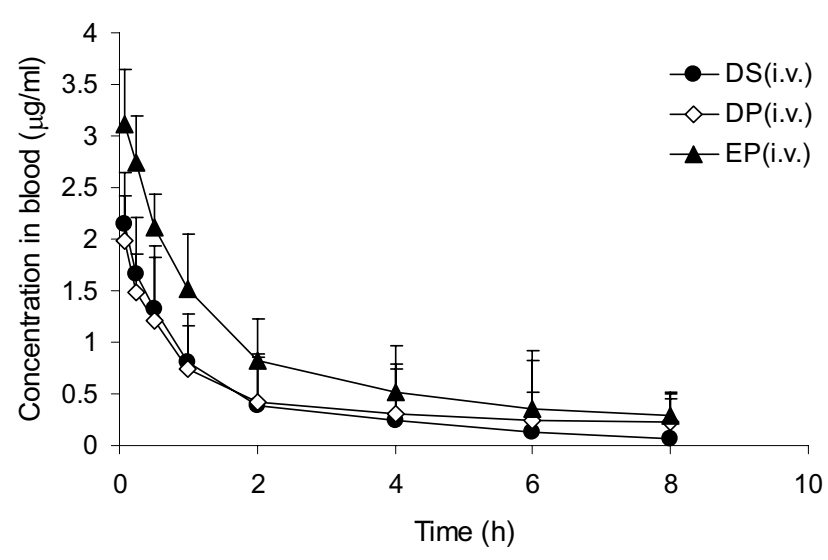

Fig. 1. Blood levels of intravenously injected Dox delivered in solution, DP and EP nanoparticles.

DS-Dox solution, DP-Dox loaded DP nanoparticles, EP-Dox loaded EP nanoparticles, i.v.-intravenous injection. The values plotted are mean $\pm \mathrm{SD}$ of three experiments. under curve ( $\mathrm{AUC}^{0-8}$ and $\mathrm{AUC}^{0-\infty}$ ) and area under moment curve $\left(\mathrm{AUMC}^{0-8}\right)$ were also higher for Dox delivered through DP and EP nanoparticles, while the clearance $(\mathrm{Cl})$ and elimination rate constant $\left(\mathrm{K}_{\mathrm{el}}\right)$ were significantly lower. The blood clearance of Dox delivered through EP nanoparticles $(0.13 \mathrm{ml} / \mathrm{min})$ was significantly lower than that delivered through DP nanoparticles $(0.21 \mathrm{ml} / \mathrm{min})$, and the $\mathrm{AUC}^{0-8}, \mathrm{AUC}^{0-\infty}$ and $\mathrm{AUMC}^{0-8}$ were significantly higher.

Fig. 2 represents the blood concentration profiles of Dox solution and Dox loaded DP and EP nanoparticles after i.p. injection in rats. The blood levels of Dox delivered through nanoparticles was relatively higher than Dox solution. The half-life $\left(\mathrm{T}_{1 / 2}\right)$ of Dox was significantly increased by DP and EP nanoparticles. The elimination rate constant of Dox delivered through nanoparticles $\left(0.2 \mathrm{~h}^{-1}\right.$ and $0.16 \mathrm{~h}^{-1}$ for DP and EP nanoparticles respectively) was lower than for Dox solution $\left(0.43 \mathrm{~h}^{-1}\right)$. The MRT of Dox delivered through DP nanoparticles was lower than for the Dox solution, while that for Dox delivered through EP nanoparticles was higher than for Dox solution. The $\mathrm{AUC}^{0-8}, \mathrm{AUC}^{0-\infty}$ and $\mathrm{AUMC}^{0-8}$ of Dox delivered through nanoparticles were significantly higher than for Dox solution. The bioavailability of Dox was greatly enhanced by DP (1.9 fold) and EP nanoparticles (2.12 fold). The bioavailability and MRT of Dox was significantly enhanced by EP nanoparticles compred to DP nanoparticles. The half-life of Dox was higher when delivered in EP nanoparticles $(4.31 \mathrm{~h})$ than in DP nanoparticles $(3.44 \mathrm{~h})$. Both $\mathrm{AUC}^{0-8}, \mathrm{AUC}^{0-\infty}$ and $\mathrm{AUMC}^{0-8}$ of Dox were significantly higher when delivered in EP nanoparticles (13.06, 20.03 and $53.78 \mathrm{~h} . \mu \mathrm{g} / \mathrm{ml}$ respectively) than in DP nanoparticles ( 11.88 and 15.01 and 40.29 h. $\mu \mathrm{g} / \mathrm{ml}$ respectively).

Fig. 3 shows the tissue concentrations of DP nanoparticles after i.v. administration in rats. The Dox delivered through nanoparticles experienced enhanced distribution to the organs of reticuloendothelial system (RES) such as

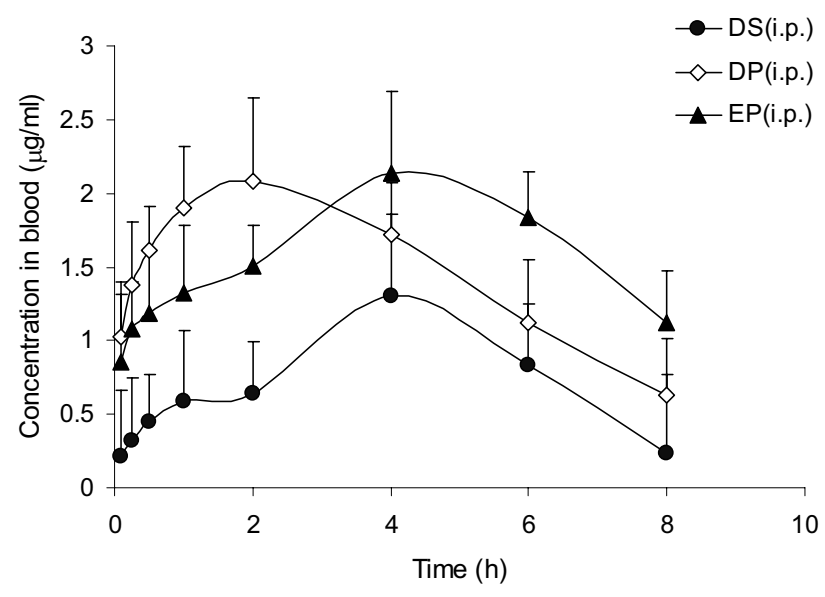

Fig. 2. Blood levels of intraperitoneally injected Dox delivered in solution, DP and EP nanoparticles. DS-Dox solution, DP-Dox loaded DP nanoparticles, EP-Dox loaded EP nanoparticles, i.p.-intraperitoneal injection. The values plotted are mean \pm SD 


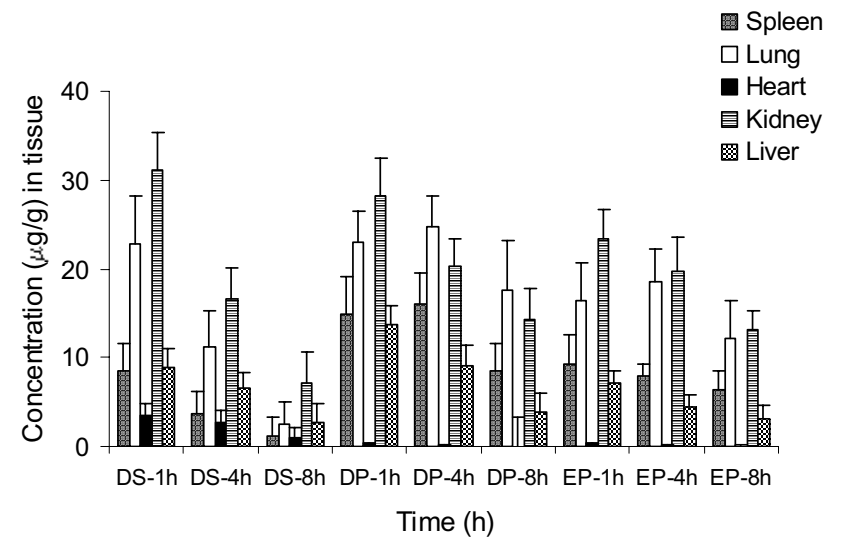

Fig. 3. Tissue distribution of Dox after intravenous injection in solution, DP nanoparticle and EP nanoparticle form.

DS-Dox solution, DP-Dox loaded DP nanoparticles, EP-Dox loaded EP nanoparticles.

The values plotted are mean \pm SD of three experiments.

spleen, liver and lung than Dox solution alone. The distribution of Dox delivered through DP nanoparticles to the organs of RES was significantly higher than that delivered through EP nanoparticles. Both types of nanoparticles significantly lowered the distribution of Dox to heart and resulted in significantly lower concentrations than Dox solution at all the time points studied.

The tissue concentrations of Dox solution and Dox delivered through nanoparticles after i.p. injection were significantly lower than after i.v. injection. After i.p. injection, the distribution of Dox delivered through DP nanoparticles was rapid to spleen and liver, while the distribution to lung increased after $1 \mathrm{~h}$ of injection. The concentration of Dox delivered through EP nanoparticles was lower in organs of RES compared to Dox solution and DP nanoparticles. The nanoparticles greatly reduced the distribution of Dox to heart.

\section{DISCUSSION}

Dox loaded poly(butylcyanoacrylate) nanoparticles were prepared by dispersion polymerization and emulsion polymerization technique. The EP technique resulted in nanoparticles with smaller size than DP, due to the high degree of compartmentalization of polymerization of polymerization locus by the added surfactant Poloxamer 188. ${ }^{9}$ The influence of polymerization factors on particle properties and drug release of EP nanoparticles were reported earlier. ${ }^{9}$ The experimental results reveal that the biodistribution of Dox is greatly altered when delivered through nanoparticles. The nanoparticles significantly enhanced the circulation half-life of Dox in blood. The initial rapid clearance of Dox loaded into DP nanoparticles is due to their ingestion by macrophages. This is fur-

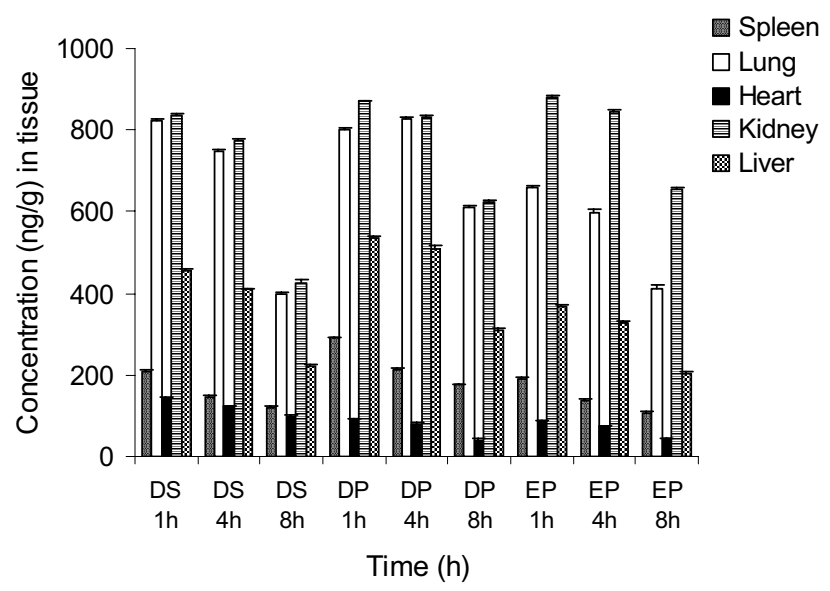

Fig. 4. Tissue distribution of Dox after intraperitoneal injection in solution, DP nanoparticle and EP nanoparticle form.

DS-Dox solution, DP-Dox loaded DP nanoparticles, EP-Dox loaded EP nanoparticles.

The values plotted are mean \pm SD of three experiments.

ther supported by the initial high concentrations of Dox delivered through DP nanoparticles in organs of RES. The EP nanoparticles resulted in higher Dox concentrations in blood than DP nanoparticles, demonstrating their long circulating characteristics. EP nanoparticles were prepared by using Poloxamer 188 as stabilizer. During the polymerization of monomer, the primary particles formed were stabilized by the surfactant Poloxamer 188 and prevents the particle coagulation by the steric repulsion mechanism. ${ }^{9}$ Polymerization in the presence of Poloxamer 188 has significantly reduced the zeta potential of nanoparticles formed, than the nanoparticles synthesized through dispersion polymerization using Dextran 70 as stabilizer. ${ }^{22}$ This is probably due to the masking of surface charge of nanoparticles by the adsorbed Poloxamer 188. The creation of hydrophilic surface at the particlewater interface by Poloxamer 188 may be a probable reason for the enhanced half-life of Dox observed with EP nanoparticles. The significantly higher $\mathrm{AUC}^{0-8}, \mathrm{AUC}^{0-\infty}$ and $\mathrm{AUMC}^{0-8}$ of Dox delivered through EP nanoparticles clearly demonstrate the long circulation property of EP nanoparticles. This is further supported by the low distribution of Dox delivered through EP nanoparticles to the tissues studied. A very important aspect is the significant reduction in distribution of Dox to heart by both DP and EP nanoparticles indicating their potential in reducing the cardiotoxicity associated with Doxorubicin therapy.

After i.p. injection, the Dox delivered through nanoparticles showed rapid appearance in blood. From blood, the DP nanoparticles were rapidly distributed to organs of RES due to their opsonization and subsequent clearance by macrophages. Interestingly, the disappearance of Dox loaded into EP nanoparticles from peritoneal cavity was slower than Dox in DP nanoparticles. Both DP and EP nanoparticles significantly increased the bioavailability of 
Table 1. Comparative pharmacokinetic parameters of Dox delivered in solution or through DP and EP nanoparticles after intravenous injection.

\begin{tabular}{|l|c|c|c|}
\hline \multicolumn{1}{|c|}{ Parameters } & DS (i.v.) & $\begin{array}{c}\text { DP } \\
\text { (i.v.) }\end{array}$ & EP (i.v.) \\
\hline $\begin{array}{l}\mathrm{K}_{\mathrm{el}}, \text { elimination } \\
\text { rate constant, } \mathrm{h}^{-1}\end{array}$ & 0.41 & $0.25^{*}$ & $0.26^{*}$ \\
$\mathrm{~T}_{1 / 2}, \mathrm{~h}$ & 1.68 & $2.74^{*}$ & $2.56^{*, \mathrm{a}}$ \\
$\mathrm{V}_{\mathrm{d}, \mathrm{ml} / \mathrm{gm}}$ & 0.71 & $0.84^{*}$ & $0.50^{*, \mathrm{a}}$ \\
$\mathrm{Cl}, \mathrm{clearance}, \mathrm{ml} / \mathrm{min}$ & 0.29 & $0.21^{*}$ & $0.13^{*, \mathrm{a}}$ \\
$\mathrm{MRT}^{\mathrm{h}} \mathrm{h}$ & 1.95 & $2.64^{*}$ & $2.41^{*, \mathrm{a}}$ \\
$\mathrm{AUC}{ }^{0-8}, \mathrm{~h} . \mu \mathrm{g} / \mathrm{ml}$ & 3.08 & $3.54^{*}$ & $6.16^{*, \mathrm{a}}$ \\
$\mathrm{AUC}^{0-\infty}, \mathrm{h} . \mu \mathrm{g} / \mathrm{ml}$ & 3.23 & 4.41 & 7.23 \\
$\mathrm{AUMC}^{0-8}, \mathrm{~h} . \mu \mathrm{g} / \mathrm{ml}$ & 6.04 & $9.39^{*}$ & $14.89^{*, \mathrm{a}}$ \\
\hline
\end{tabular}

*extremely significant difference between DS (i.v.) vs DP (i.v.) and EP (i.v.). *indication is given to the respective data. (Significance level $\mathrm{P}<0.001$ )

${ }^{a}$ extremely significant difference between DP (i.v.) and EP (i.v.). (Significance level $\mathrm{P}<0.001$ )

DS-Dox solution, DP-Dox loaded DP nanoparticles, EP-Dox loaded EP nanoparticles, i.v.-intravenous injection.

Table 2. Comparative pharmacokinetic parameters of Dox delivered in solution or through DP and EP nanoparticles after intraperitoneal injection.

\begin{tabular}{|c|c|c|c|}
\hline Parameters & $\begin{array}{l}\text { DS } \\
\text { (i.p.) }\end{array}$ & $\begin{array}{l}\text { DP } \\
\text { (i.p.) }\end{array}$ & EP (i.p.) \\
\hline $\begin{array}{l}\mathrm{K}_{\mathrm{el}} \text {, elimination rate } \\
\text { constant, } \mathrm{h}^{-1}\end{array}$ & 0.43 & $0.20 *$ & $0.16^{*, \mathrm{a}}$ \\
\hline $\mathrm{T}_{1 / 2}, \mathrm{~h}$ & 1.60 & $3.44 *$ & $4.31^{*, \mathrm{a}}$ \\
\hline $\mathrm{F}, \mathrm{h} . \mu \mathrm{g} / \mathrm{ml}$ & 1.99 & $3.84 *$ & $4.22 * a \mathrm{a}$ \\
\hline $\mathrm{V}_{\mathrm{d},} \mathrm{ml} / \mathrm{gm}$ & 0.74 & $1.61^{*}$ & $2.01^{*, \mathrm{a}}$ \\
\hline $\mathrm{Cl}$, clearance, $\mathrm{ml} / \mathrm{min}$ & 0.32 & 0.32 & 0.32 \\
\hline MRT, h & 4.00 & $3.39 *$ & $4.11^{*, \mathrm{a}}$ \\
\hline $\mathrm{AUC}^{0-8}, \mathrm{~h} . \mu \mathrm{g} / \mathrm{ml}$ & 6.15 & $11.88 *$ & $13.06^{*, \mathrm{a}}$ \\
\hline $\mathrm{AUC}^{0-\infty}, \mathrm{h} \cdot \mu \mathrm{g} / \mathrm{ml}$ & 6.68 & 15.01 & 20.03 \\
\hline $\mathrm{AUMC}^{0-8}, \mathrm{~h} . \mu \mathrm{g} / \mathrm{ml}$ & 24.69 & $40.29 *$ & $53.78^{*, \mathrm{a}}$ \\
\hline
\end{tabular}

*extremely significant difference between DS (i.p.) vs DP (i.p.) and EP (i.p.). *indication is given to the respective data. (Significance level $\mathrm{P}<0.001$ )

${ }^{a}$ extremely significant difference between DP (i.p.) and EP (i.p.). (Significance level $P<0.001$ )

DS-Dox solution, DP-Dox loaded DP nanoparticles, EP-Dox loaded EP nanoparticles, i.p.-intraperitoneal injection.
Dox administered intraperitoneally. However, the bioavailability enhancement of Dox was more pronounced by EP nanoparticles than DP nanoparticles. The rapid appearance of nanoparticle delivered Dox in blood is probably due to the smaller size of nanoparticles facilitating quick absorption into blood vessels. The lower bioavailability of DP nanoparticle its delivered Dox may be due to their uptake in small amounts by peritoneal macrophages. The lower $\mathrm{K}_{\mathrm{el}}$ of Dox delivered through nanoparticles compared to Dox solution indicates their slow clearance from the body, which is supported by the enhanced half-life and $\mathrm{AUC}^{0-8}, \mathrm{AUC}^{0-\infty}$ and $\mathrm{AUMC}^{0-8}$. The pharmacokinetic data clearly signifies the advantageous role of EP nanoparticles in enhancing the circulation time of Dox in blood. The nanoparticles decreased the distribution of Dox to heart suggesting their advantage in reducing the cardiotoxicity associated with Dox therapy. The slow and prolonged clearance of EP nanoparticles from peritoneal cavity into blood can be useful in local chemotherapy of peritoneal tumors, wherein the tumor cells are exposed to the nanoparticles containing Dox for longer time and are expected to result in greater antitumor activity than with the therapy by i.v. administration.

\section{CONCLUSION}

Incorporation of Dox into nanoparticles has greatly altered its biodistribution. DP nanoparticles resulted in rapid clearance of Dox from blood and facilitated distribution to the organs of RES. EP nanoparticles enhanced the circulation half-life of Dox in blood and also reduced its tissue distribution. The long circulation property of EP nanoparticles due to their smaller size could be dox delivering explained to tumors by EPR (enhanced permeability and retention) effect. The slow and prolonged clearance of Dox delivered through EP nanoparticles from peritoneal cavity may be advantageous in the local chemotherapy of peritoneal tumors. Both DP and EP nanoparticles showed significant enhancement of bioavailability of Dox compared to Dox solution after i.p. injection indicating the advantage of nanoparticles in improving the blood concentrations after i.p. injection. The study demonstrates the potential use of EP nanoparticle loaded Dox in improving the therapy with Dox and reduce the dox-associated systemic toxicity.

\section{ACKNOWLEDGEMENT}

Financial support from the University Grants Commission (F.10-32/2000 (SA-II)) New Delhi, India is gratefully acknowledged. 


\section{REFERENCES}

1. Kreuter J. Nanoparticles. In: Kreuter J editor. Colloidal drug delivery systems, Marcel Dekker, New York, 1994. p. 219-342.

2. Troster SD, Muller U, Kreuter J. (1990) Modification of the body distribution of poly(methyl methacrylate) nanoparticles in rats by coating with surfactants. Int J Pharm 61, 85-100.

3. Grislain L, Couvreur P, Lenaerts V, Roland M, Deprez-Decampeneere D, Speiser P. (1983) Pharmacokinetics and biodistribution of a biodegradable drug-carrier. Int J Pharm 15, 335-345.

4. Lowe PJ, Temple CS. (1994) Calcitonin and insulin in isobutyl cyanoacrylate nanocapsules: protection against proteases and effect of intestinal absorption in rats. J Pharm Pharmacol 46, 547-552.

5. Verdun C, Couvreur P, Vranckx H, Lenaerts V, Roland M. (1986) Development of a nanoparticle controlled-release formulation for human use. J Cont Rel 3, 205-210.

6. Sommerfeld P, Schroeder U, Sabel BA. (1997) Long-term stability of PBC nanoparticle suspensions suggests clinical usefulness. Int J Pharm 155, 201-207.

7. Douglas SJ, Illum L, Davis SS, Kreuter J. (1984) Particle size and size distribution of poly(butyl-2-cyanoacrylate) nanoparticles I. Influence of physicochemical factors. J Colloid Interface Sci 101 149-158.

8. Douglas SJ, Davis SS, Illum L. (1986) Biodistribution of poly(butyl 2-cyanoacrylate) nanoparticles in rabbits. Int $\mathbf{J}$ Pharm 34, $145-$ 152

9. Harivardhan RL. Murthy RSR. (2003) Polymerization of n-butyl cyanoacrylate in presence of surfactant: study of influence of polymerization factors on particle properties, drug loading and evaluation of its drug release kinetics. Ars Pharmaceutica 44, 351-369.

10. Proffitt RT, Williams LE, Presant CA, Tin GW, Ulina JA, Gamble RC, Baldeschwieler JD. (1983) Liposomal blockade of the reticuloendothelial system: improved tumor imaging with small unilamellar vesicles. Science 220, 502-505.

11. Illum L, Davis SS. (1984) The organ uptake of intravenously administered colloidal particles can be altered using a non-ionic surfactant (Poloxamer 338). FEBS Lett 167, 79-82.
12. Lue D, Manthey B, Kreuter J, Speiser P, Deluca PP. (1984) Distribution and elimination of coated polymethyl $\left[2-{ }^{14} \mathrm{C}\right)$ methacrylate nanoparticles after intravenous injection in rats. J Pharm Sci 73, 1433-1437.

13. Storm G, Belliot SO, Daemen T, Lasic DD. (1995) Surface modification of nanoparticles to oppose uptake by the mononuclear phagocytic system. Adv Drug Del Rev 17, 31-48.

14. Gulyaev AE, Gelperina SE, Skidan IN, Antropov AS, Kivman, GY, Kreuter J. (1999) Significant transport of doxorubicin into the brain with polysorbate 80 -coated nanoparticles. Pharm. Res 16, 1564-1569.

15. Peracchia MT, Fattal E, Desmaele D, Besnard M, Noel JP, Gomis JM, Appel M, d'Angelo J, Couvreur P. (1999) Stealth PEGylated polycyanoacrylate nanoparticles for intravenous administration and splenic targeting. J Cont Rel 60, 121-128.

16. Dembri A, Montisci M-J, Gantier jc, Chacun H, Ponchel G. (2001) Targeting of 3'-Azido3'-Deoxythymidine (AZT)-loaded Poly(isohe xylcyanoacrylate) nanospheres to the gastrointestinal mucosa and associated lymphoid tissues. Pharm Res 18, 467-473.

17. Maeda H, Wu J, SAWA T, Matsumura y, Hori K. (2000) Tumor vascular permeability and the EPR effect in macromolecular therapeutics: a review. J Cont Rel 65, 271-284.

18. Jain RK. (2001) Delivery of molecular medicine to solid tumors: lessons from in vivo imaging of gene expression and function. J Cont Rel 74, 7-25.

19. Duncan R. (1999) Polymer conjugates for tumor targeting and intracytoplasmic delivery. The EPR effect as a common gateway? Pharm Sci Tech Today 2, 441-249.

20. Vigevani A, Williamson MJ. Doxorubicin. In: Florey K editor. Analytical profiles of drug substances. Academic Press, New York, 1980. p. 245-274.

21. Reddy L.H. Murthy RSR. Fluorimetric estimation of Doxorubicin hydrochloride in plasma, whole blood and tissues of rat. Communicated.

22. Reddy L.H. Murthy RSR. (2004) Influence of polymerization technique and experimental variables on particle properties and release kinetics of methotrexate from PBC nanoparticles. Acta Pharm In Press. 